\title{
Prehospital identification of Covid-19: an observational study
}

\author{
Douglas Spangler ${ }^{*}$, Hans Blomberg and David Smekal
}

\begin{abstract}
Background: The novel coronavirus disease 2019 (Covid-19) pandemic has affected prehospital care systems across the world, but the prehospital presentation of affected patients and the extent to which prehospital care providers are able to identify them is not well characterized. In this study, we describe the presentation of Covid-19 patients in a Swedish prehospital care system, and asses the predictive value of Covid-19 suspicion as documented by dispatch and ambulance nurses.

Methods: Data for all patients with dispatch, ambulance, and hospital records between January 1-August 31, 2020 were extracted. A descriptive statistical analysis of patients with and without hospital-confirmed Covid-19 was performed. In a subset of records beginning from April 14, we assessed the sensitivity and specificity of documented Covid-19 suspicion in dispatch and ambulance patient care records.
\end{abstract}

Results: A total of 11,894 prehospital records were included, of which 481 had a primary hospital diagnosis code related to-, or positive test results for Covid-19. Covid-19-positive patients had considerably worse outcomes than patients with negative test results, with 30 -day mortality rates of $24 \%$ vs $11 \%$, but lower levels of prehospital acuity (e.g. emergent transport rates of $14 \%$ vs $22 \%$ ). About half (46\%) of Covid-19-positive patients presented to dispatchers with primary complaints typically associated with Covid-19. Six thousand seven hundred seventy-six records were included in the assessment of predictive value. Sensitivity was 76\% (95\% Cl 71-80) and 82\% (78-86) for dispatch and ambulance suspicion respectively, while specificities were $86 \%(85-87)$ and $78 \%(77-79)$.

Conclusions: While prehospital suspicion was strongly indicative of hospital-confirmed Covid-19, based on the sensitivity identified in this study, prehospital suspicion should not be relied upon as a single factor to rule out the need for isolation precautions. The data provided may be used to develop improved guidelines for identifying Covid-19 patients in the prehospital setting.

\section{Introduction}

\section{Background}

The pandemic of novel coronavirus disease 2019 (Covid19) caused by Severe Acute Respiratory Syndrome Coronavirus 2 (SARS-CoV-2) has impacted healthcare systems across the world, and much research has been done regarding the underlying mechanisms of the disease [1], the clinical presentation of affected patients [2,

\footnotetext{
* Correspondence: douglas.spangler@akademiska.se

Uppsala Center for Prehospital Research, Department of Surgical Sciences Anesthesiology and Intensive care, Uppsala University, 75185 Uppsala, Sweden
}

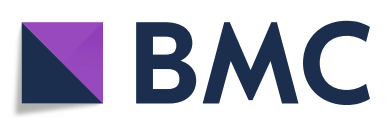

$3]$, prevention and treatment methodologies [4, 5], and the impact on population-level health [6]. Prehospital care providers play an important role in initiating disease isolation precautions and are instrumental in preventing contagion at ambulance receiving facilities. If prehospital care providers identify Covid-19 patients and document their suspicion with a high degree of precision, this documentation could be used as the basis for determining the need for isolation precautions on the ambulance and at receiving facilities.

Despite the significant Covid-19 related scientific output [7], only a few studies describing the effect of the

(c) The Author(s). 2020 Open Access This article is licensed under a Creative Commons Attribution 4.0 International License, which permits use, sharing, adaptation, distribution and reproduction in any medium or format, as long as you give appropriate credit to the original author(s) and the source, provide a link to the Creative Commons licence, and indicate if changes were made. The images or other third party material in this article are included in the article's Creative Commons licence, unless indicated otherwise in a credit line to the material. If material is not included in the article's Creative Commons licence and your intended use is not permitted by statutory regulation or exceeds the permitted use, you will need to obtain permission directly from the copyright holder. To view a copy of this licence, visit http://creativecommons.org/licenses/by/4.0/ The Creative Commons Public Domain Dedication waiver (http://creativecommons.org/publicdomain/zero/1.0/) applies to the data made available in this article, unless otherwise stated in a credit line to the data. 
Covid-19 pandemic on prehospital care systems are available [8-10], and these are largely limited to reports on the impact of the pandemic at the system-level. Only a single study has been published which quantitatively investigates the characteristics of Covid-19 patients and/ or the ability of prehospital care providers to identify SARS-CoV-2-positive patients by linking prehospital data with subsequent hospital diagnoses [11]. In this study, Fernandez et al. found that Covid-19 patients more often presented to prehospital care providers with tachycardia, tachypnea, hypoxia, and fever, and found a sensitivity of $78 \%$ for ambulance crews in identifying confirmed Covid-19 patients based on an analysis of structured documentation and free text notes.

\section{Objective}

In this study, we sought to describe the clinical presentation of Covid-19 patients in relation to appropriate comparison cohorts, and to assess the ability of prehospital care providers to identify Covid-19 patients the dispatch center and on the ambulance.

\section{Setting}

The region of Uppsala, Sweden has an area of $8209 \mathrm{~km} 2$, and a population of 385,000 . The region is served by one major university hospital and a smaller regional hospital, an ambulance service with 13/18 (night-time/daytime) ambulances, and a single regional Emergency Medical Dispatch (EMD) center. Ambulances are staffed by either two nurses, or one nurse and an emergency medical technician. Call-takers at the dispatch center are exclusively nurses, with 3-4 years of university education and a minimum of 3 years of clinical experience.

At the peak of the pandemic, the Emergency Medical Dispatch (EMD) center experienced a ca. 10-20\% increase in call volume. The guidelines employed by the ambulance service and dispatch center to identify Covid19 patients evolved over time as knowledge about the disease increased. The first guidelines regarding Covid19 suspicion were implemented on February 28, 2020, setting the criteria for Covid-19 suspicion as the presence of a fever and respiratory symptoms (e.g., coughing or difficulty breathing), and having within the previous 14 days visited China, Italy, Iran, or South Korea, or been in contact with a person being evaluated for Covid-19. By March 13, community transmission of Covid-19 had been detected in Sweden, and the criterion based on previous travel was dropped. On April 1, guidelines were updated such that any one of four symptoms (Fever, cough, difficulty breathing, upper airway symptoms) was sufficient to trigger Covid-19 precautions. On May 7, diarrhea was added to the list of symptoms which would trigger Covid-19 suspicion.
The ability to document suspicion of Covid-19 in a structured format was added first to the dispatch clinical decision support system on March 19, and then to the ambulance's electronic patient care reporting system on April 14. Prior to these dates, suspicion of Covid-19 was generally documented in free-text notes and/or communicated between care providers verbally via radio, telephone, or at patient handover.

\section{Methods}

\section{Data sources}

Data were extracted from dispatch, ambulance, and hospital records in the region of Uppsala, Sweden, and deterministically linked based on patient personal identification number and case identifier for the period of January 1August 31, 2020. All single-patient EMD center contacts which could be linked to both an ambulance record and a hospital record were included in the study. For calls where the patient was evaluated but not transported by the ambulance, hospital records were searched, and patients visiting a healthcare facility within $72 \mathrm{~h}$ of contact with the prehospital care system were included in the analysis.

\section{Variables}

Covid-19 patients were identified based on documentation of either a real-time reverse transcription positive polymerase chain reaction (PCR)-based test for an ongoing SARS-CoV-2 infection at any point during the first hospital care episode following contact with the ambulance, or a primary diagnosis code relating to Covid-19 (ICD-10SE codes U07.1, U07.2). Samples for PCR testing were obtained using the combined oro-nasopharyngeal swabbing technique.

For the purposes of the descriptive analysis, patient cohorts were generated consisting of patients cared for prior to the beginning of the Covid-19 pandemic in Uppsala (January 1 - February 29), patients cared for at the hospital but not tested for SARS-CoV-2 ("Assumed negative"), patients tested and found negative for SARSCoV-2 ("Confirmed negative"), and patients who tested positive or were otherwise diagnosed with Covid-19 ("Positive").

Hospital outcome measures included rates of hospital admission, intensive care utilization, and 30-day mortality. Covariates extracted from dispatch and ambulance records for analysis included patient demographics (age and gender), response and transport priority (Lights and sirens vs. no lights and sirens), and initial ambulance findings (Airway/Breathing/Circulation assessments), vital signs collected by the ambulance (pulse, systolic blood pressure, breathing rate, Spo2, and temperature), and interventions performed by ambulance crews (Oxygen/medication administration). We included a variable indicating the primary complaint of the patient (as 
categorized by the dispatch nurse), and based on local guidelines, considered the complaints of infection, fever, difficulty breathing, and upper airway distress to be "typical" of Covid-19.

\section{Statistical methods}

A time-series analysis was performed to investigate the impact of Covid-19 on the prehospital care system in the region over time, using local polynomial regression fitting (loess) on a back-transformed $\log (x+1)$ scale to enable estimating rolling mean values at- and/or close to zero [12]. Statistics regarding means, medians, and percentages were generated as appropriate, using ordinary non-parametric bootstrapping to generate $95 \%$ confidence intervals [13]. Continuous variables with non-monotonic associations with Covid-19 status (e.g. vital signs) were described using kernel density plots. Missing vital sign data, and values above and below the 99.9th percentile were excluded from each respective plot in order to eliminate values stemming from documentation errors.

The accuracy of documented Covid-19 suspicion was assessed using standard epidemiological test statistics including sensitivity, specificity, and negative/positive predictive value. Confidence intervals for test statistics were calculated using exact binomial tests as implemented in the epiR package $[14,15]$. Longitudinal shifts in predictive value were assessed by plotting each measure grouped by month. Given the potential for bias due to missed cases of Covid-19 among assumed negative patients, we analyzed statistics based both on the assumption that untested patients were negative per the study by Fernandez et al. [11], and a sensitivity analysis investigating only PCR-test results. All analyses were performed using $\mathrm{R}$ version 4.0.2 [16]. Results were reported based on the STROBE guidelines [17].

\section{Results \\ Descriptive data}

As described in Fig. 1, a total of 38,654 records were identified. Of these, 25,917 had a medical problem, with the remainder being either administrative assignments (e.g. stand-by or test records) or misdirected calls (e.g. police/fire calls or accidental calls). Of these, 16,464 were determined by a dispatch nurse to require an ambulance, with the remainder of patients referred to alternate forms of care (e.g. alternate forms of transport to definitive care, the nursing advice line, or to self-care pending a change in symptoms). Of these, 15,622 had both a documented personal identification number and a linked ambulance record. Finally, hospital records could be identified for 11,775 of these patients, with the remainder having either been treated and released onscene (and did not visit a hospital-based care provider within $72 \mathrm{~h}$ ), or had a misregistered personal identification number resulting in a linkage failure. Thus, a total of 11,775 hospital visits with linked prehospital data were identified over the study period.

Of the patients included, 2684 (23\%) were tested for ongoing SARS-CoV-2 infection via PCR, of whom 356 (13\%) were found to be positive at any point during the hospital episode. A total of 404 patients had a primary diagnosis code indicating Covid-19, of whom 125 were diagnosed with Covid-19 without a positive test (potentially due to the results of tests performed outside of the hospital, or via other means of diagnosis including radiology). An additional 77 patients were found to have a positive test result with no corresponding diagnosis, for a total of 481 "hospital-confirmed" cases of Covid-19.

Of the included records, 6794 occurred on or after April 14 and were included in the analysis of prehospital predictive value. Of 2252 PCR tests were performed following this date, 279 were positive. An additional 77 Covid-19 patients were identified via diagnosis code, for a total of 481 hospital confirmed cases during this time frame.

A total of 1381 patients had suspicion of Covid-19 documented by the dispatch center, while 1690 had suspicion documented in the ambulance journal. The volume of these patients over time is reported in Fig. 2 below.

Table 1 below presents descriptive statistics regarding the study cohort, divided into comparison groups.

Covid-19 patients had a 30 -day mortality rate of $23.7 \%$ (95\% CI 19.8-27.4), and 16.2\% (12.9-19.5) were admitted to an intensive care unit. $30.1 \%(25.8-34.3)$ of Covid-19 patients received a high priority ambulance from dispatch, while $14.1 \%$ (11-17.5) were transported by the ambulance with a high priority. $36.4 \%(31.8-41.0)$ received supplemental oxygen, while $28.5 \%$ (24.7-32.4) received other medications administered by ambulance crews. The median mission time (from receipt of call at the dispatch center to ambulance clearance from the hospital, including time spent sanitizing the ambulance) was 128 (123-132) minutes.

Table 2 presents the distribution of Covid-19 suspicion and test results across call types documented by the EMD center. A total of 1223 patients (18\%, including 27 patients with an upper airway-related complaint) contacted the dispatch center with a primary complaint directly related to the Covid-19 suspicion guidelines, while 222 (46\%) confirmed Covid-19 cases were among these callers.

Figure 3 presents density functions for the initial vital signs of patients in each of the comparison groups. In comparing Covid-19-positive and patients tested negative, all vital signs except for pulse rates demonstrated statistically significant differences in central tendency per Wilcoxon rank sum tests. Body temperature demonstrated the most substantial effects, with $23 \%$ of patients with a negative Covid-19 test result presenting with a 


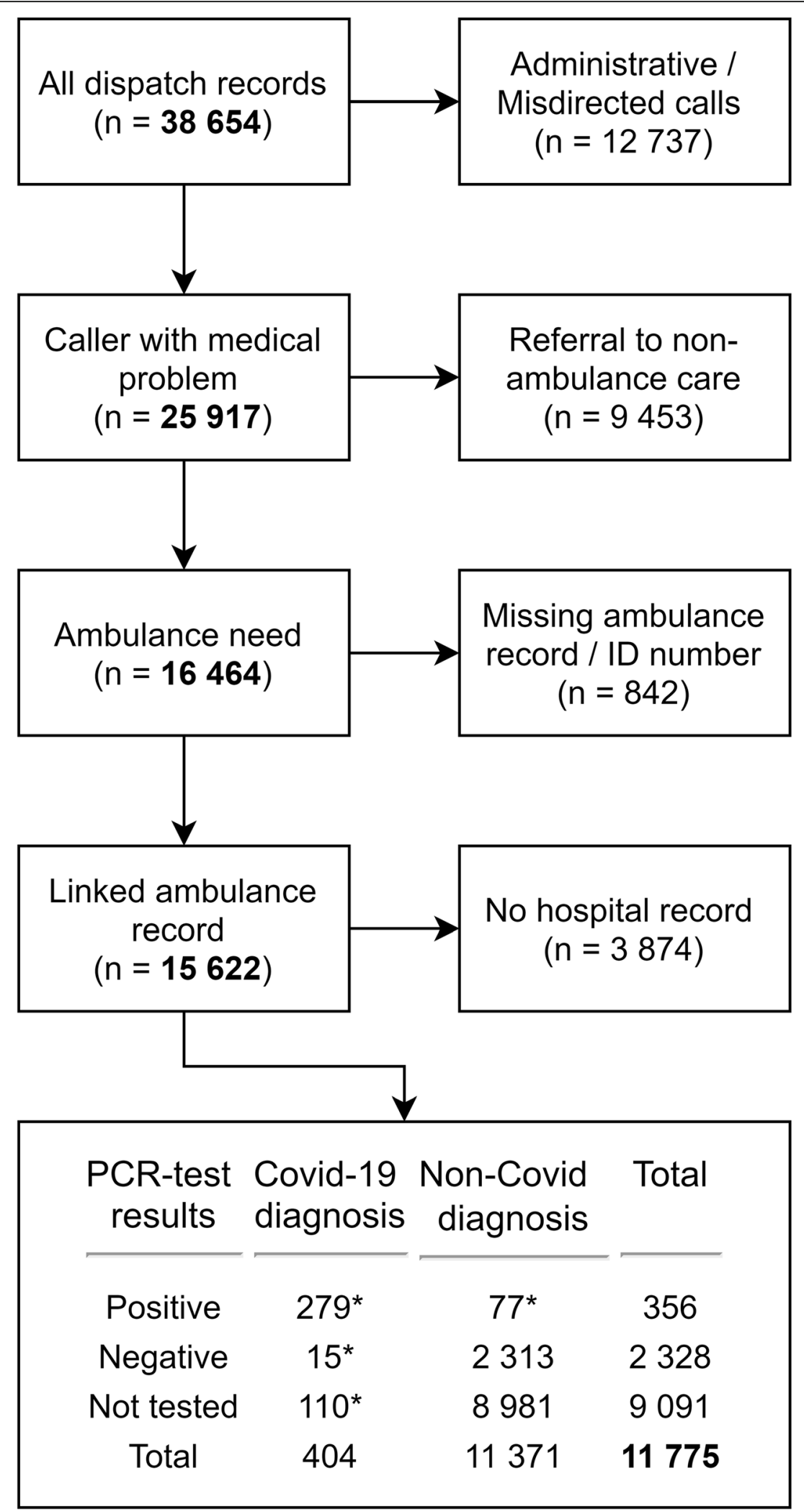

Fig. 1 Inclusion flow chart.* Patients considered "hospital-confirmed" Covid-19 


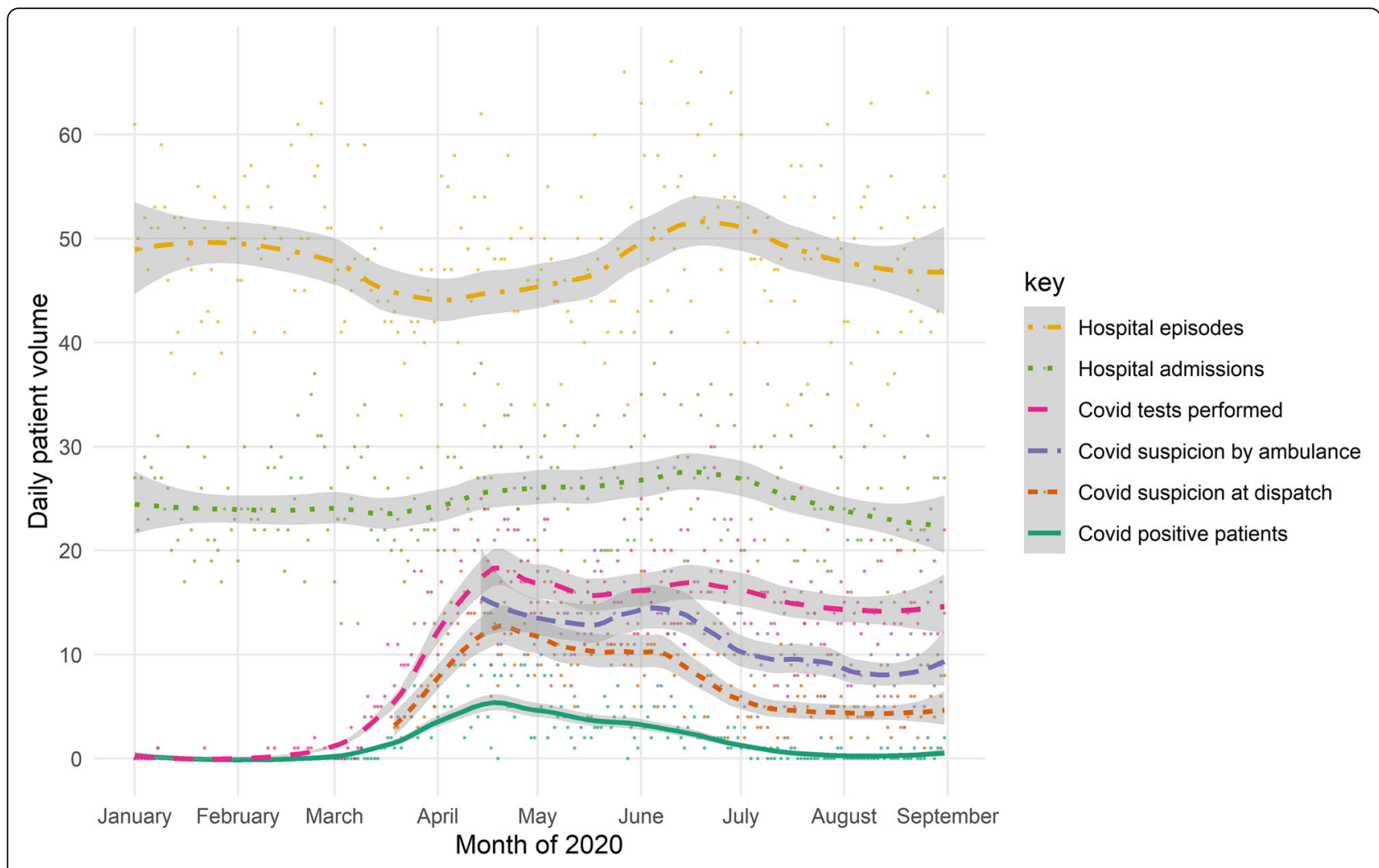

Fig. 2 Absolute daily patient volumes (individual points) over study period with smoothed average rates with 95\% confidence intervals (shaded area). Rolling averages for dispatch and ambulance suspicion are plotted from the dates they were implemented (March 19 and April 14, respectively)

Table 1 Patient demographics and clinical characteristics

\begin{tabular}{lllll}
\hline $\mathbf{N}$ & Pre-Covid & Assumed negative & Tested negative & Positive \\
& $\mathbf{2 9 9 8}$ & $\mathbf{5 9 8 3}$ & $\mathbf{2 3 1 3}$ & $\mathbf{4 8 1}$ \\
\hline Female, \% & $52.8(51.0-54.6)$ & $51.2(50.1-52.5)$ & $50.2(48.2-52.3)$ & $47.0(42.2-51.1)$ \\
Age, median & $70.0(70.0-71.0)$ & $65.0(64.0-66.0)$ & $77.0(76.0-77.0)$ & $73.0(69.0-74.0)$ \\
High priority dispatch, \% & $48.1(46.3-49.9)$ & $46.7(45.4-48.0)$ & $40.9(38.9-42.8)$ & $30.1(25.8-34.3)$ \\
Abnormal airway, \% & $2.9(2.3-3.5)$ & $1.9(1.5-2.3)$ & $3.7(3.0-4.5)$ & $1.3(0.4-2.4)$ \\
Abnormal breathing, \% & $17.0(15.7-18.4)$ & $9.6(8.8-10.3)$ & $32.4(30.5-34.4)$ & $46.7(42.3-51.2)$ \\
Abnormal circulation, \% & $16.8(15.4-18.1)$ & $12.6(11.7-13.4)$ & $25.3(23.4-27.0)$ & $26.0(21.8-30.2)$ \\
Supplemental oxygen, \% & $15.3(14.0-16.6)$ & $7.2(6.5-7.8)$ & $29.5(27.5-31.3)$ & $36.4(31.8-41.0)$ \\
Medication administration, \% & $38.6(36.9-40.3)$ & $32.3(31.1-33.5)$ & $38.3(36.4-40.3)$ & $28.5(24.7-32.4)$ \\
High priority transport, \% & $15.4(14.2-16.7)$ & $11.0(10.2-11.9)$ & $22.0(20.0-23.8)$ & $14.1(11.0-17.5)$ \\
Total mission duration (minutes), median & $104(102-105)$ & $102(101-103)$ & $120(118-122)$ & $128(123-132)$ \\
Admitted to hospital, \% & $49.3(47.4-51.1)$ & $37.6(36.4-38.8)$ & $88.6(87.2-89.9)$ & $83.4(80.0-86.7)$ \\
Admitted to Intensive Care Unit, \% & $2.0(1.5-2.6)$ & $0.6(0.4-0.8)$ & $3.9(3.1-4.7)$ & $11.3(9.9-12.6)$ \\
30-day mortality, \% & $5.9(5.1-6.7)$ & $3.4(3.0-3.8)$ & $23.7(19.8-27.4)$ \\
\hline
\end{tabular}

All data presented with point estimates and bootstrapped $95 \%$ Confidence intervals. Grouped by patients cared for prior to the outbreak of Covid-19 from Jan 1Feb 29 (Pre-Covid), those not tested for Covid-19 (Assumed negative), tested negative (Tested negative), and positive for Covid-19 per PCR-test and/or primary diagnosis code (Positive) 
Table 2 Covid-19 suspicion and testing rates by primary complaint at dispatch with more than 100 occurrences from April 14 onwards, ordered by descending proportion of confirmed Covid-19 cases

\begin{tabular}{|c|c|c|c|c|c|}
\hline Primary Complaint & $\mathrm{n}$ & $\begin{array}{l}\text { Suspicion at dispatch, } \\
\%\end{array}$ & $\begin{array}{l}\text { Suspicion by ambulance, } \\
\%\end{array}$ & $\begin{array}{l}\text { Tested at hospital, } \\
\%\end{array}$ & $\begin{array}{l}\text { Hospital-confirmed a Covid-19, } \\
\%\end{array}$ \\
\hline Infection & 261 & $68.6(62.8-74.3)$ & $78.5(73.9-83.5)$ & $72.8(67.4-78.2)$ & $29.1(23.8-34.9)$ \\
\hline Fever & 147 & $71.4(64.6-79.6)$ & $76.9(70.1-83.7)$ & $74.1(67.3-81.0)$ & $16.3(10.9-22.4)$ \\
\hline Difficulty Breathing & 790 & $50.9(47.5-54.3)$ & $65.4(61.8-68.5)$ & $62.9(59.9-66.1)$ & $14.7(12.3-17.1)$ \\
\hline General Elderly ${ }^{\mathrm{b}}$ & 344 & $17.2(13.4-21.5)$ & $29.1(24.1-33.7)$ & $54.7(49.1-60.2)$ & $6.4(3.8-9.0)$ \\
\hline General Adult $^{\mathrm{b}}$ & 139 & $28.8(21.6-36.0)$ & $29.5(22.3-37.4)$ & $38.1(30.2-46.0)$ & $5.8(2.2-10.1)$ \\
\hline $\begin{array}{l}\text { Reduced } \\
\text { consciousness }\end{array}$ & 262 & $12.6(8.8-16.4)$ & $22.9(18.3-27.9)$ & $38.9(33.2-45.0)$ & $4.6(2.3-7.3)$ \\
\hline Fainting & 117 & $12.0(6.8-17.9)$ & $19.7(12.8-27.4)$ & $22.2(14.5-29.9)$ & $3.4(0.9-6.8)$ \\
\hline Stroke & 428 & $5.4(3.5-7.5)$ & $14.7(11.4-18.0)$ & $35.3(30.8-40.2)$ & $3.3(1.9-5.1)$ \\
\hline Seizure & 207 & $4.3(1.9-7.2)$ & $10.6(6.8-15.4)$ & $20.3(15.0-25.6)$ & $2.4(0.5-4.8)$ \\
\hline Other & 908 & $11.3(9.1-13.4)$ & $15.7(13.4-18.1)$ & $24.1(21.3-27.1)$ & $2.2(1.3-3.2)$ \\
\hline Dizziness & 144 & $6.9(3.5-11.8)$ & $14.6(9.0-20.8)$ & $17.4(11.1-23.6)$ & $2.1(0.0-4.2)$ \\
\hline Major trauma & 1203 & $2.8(1.9-3.8)$ & $8.1(6.6-9.7)$ & 26.4 (23.9-28.9) & $1.8(1.1-2.7)$ \\
\hline Missing & 118 & $0.0(0.0-0.0)$ & $11.0(5.9-16.9)$ & $23.7(16.1-31.4)$ & $1.7(0.0-4.2)$ \\
\hline Abdominal pain & 548 & $8.4(6.2-10.9)$ & $17.2(13.9-20.4)$ & $17.9(14.6-21.2)$ & $1.5(0.5-2.6)$ \\
\hline Chest pain & 813 & $9.5(7.4-11.4)$ & $17.7(15.3-20.5)$ & $19.7(17.1-22.4)$ & $1.2(0.5-2.1)$ \\
\hline Arrythmia & 153 & $3.3(0.7-6.5)$ & $14.4(9.2-20.3)$ & $11.8(6.5-17.0)$ & $0.7(0.0-2.6)$ \\
\hline
\end{tabular}

All data presented with point estimates and bootstrapped $95 \%$ Confidence intervals

${ }^{\text {a }}$ Confirmed based on either a positive PCR-test or by hospital diagnosis code

${ }^{\mathrm{b}}$ These categories capture patients with non-specific complaints that cannot be further categorized

fever of $>=38$ degrees centigrade, and $51 \%$ of Covid- 19 positive patients presenting with this degree of fever upon contact with the ambulance.

\section{Prehospital assessments}

Table 3 below presents the overall predictive values for dispatch and ambulance suspicion of Covid-19. Given the risk of misclassification-bias due to low testing rates, data are presented both for the full cohort of patients, and for only those with a documented PCR-test for SARS-CoV-2. The prevalence of SARS-CoV-2 in the full population was 5 , and $11 \%$ among tested patients. The apparent prevalence of Covid-19 (i.e., the percentage of patients with documented suspicion) in the full population was $17 \%$ at the dispatch, and $25 \%$ in the ambulance. Apparent prevalence among tested patients was 32 and $49 \%$ at the dispatch and ambulance, respectively. In the full population, the sensitivity of documented Covid-19 suspicion at dispatch was $75.9 \%(71.0-80.3)$ with a specificity of $86.4 \%(85.5-87.2)$ in the full cohort. The sensitivity of the ambulance suspicion was $82.2 \%$ (77.8-86.1), with a specificity of $78.2 \%$ (77.2-79.2). Levels of specificity and negative predictive value were lower within the cohort of tested patients only.

Figure 4 presents this data per study month. Over time, both the apparent and true prevalence of Covid-19 declined (Note that structured documentation on the ambulance was implemented at the peak of the pandemic). Specificity and negative predictive value increased while positive predictive value declined substantially over time. Between April and May, dispatch sensitivity changed from $75 \%(65-83)$ to $82 \%$ (74-88), while ambulance sensitivity changed from $77 \%(67-84)$ to $89 \%(83-95)$, while in later months, the scarcity of true positive cases resulted in wide confidence intervals (Fig. 4).

\section{Discussion}

\section{Key results}

In this study, we found that the $76 \%$ of hospitalconfirmed Covid-19 patients were identified by dispatch nurses, while $82 \%$ were identified by ambulance nurses. We identified a relatively high 30 -day mortality rate of $24 \%$ among Covid-19 patients, but a relatively low rate of emergent response (30\%) and transport (14\%). Covid19 patients presented with a diverse set of complaints, and a majority of Covid-19 patients (54\%) presented with primary complaints not typical of Covid-19. With the exception of body temperature, initial vital signs had little predictive value for the identification of Covid-19 patients.

\section{Limitations}

This retrospective analysis has several important limitations. Documentation completeness issues likely entail 


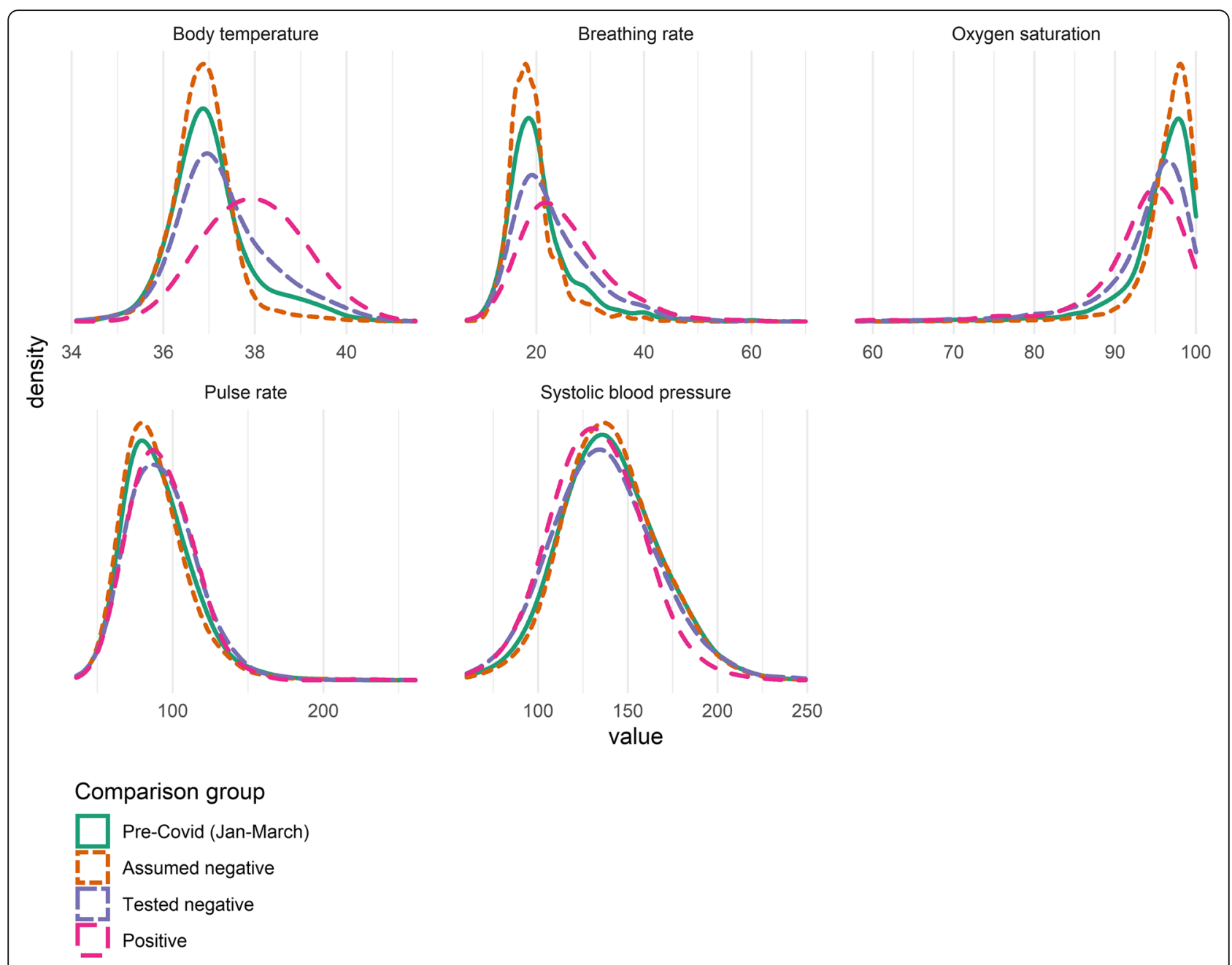

Fig. 3 Distribution of vital signs of Covid-19 patients in relation to comparison cohorts. Note: Distribution functions have no interpretable Y-axis, and it is thus left blank. Rather, the area under each curve equals 1, and the height of the curve is inversely proportional to the variance in the data

an under-estimation of the true sensitivity of prehospital suspicion of Covid-19, given that suspicion is often likely to be communicated upon handover between care providers verbally, or in free-text notes. We chose to limit this analysis to structured documentation (i.e., the selection pre-defined options) however, as only this form of data has the potential to be used in automated alerting and monitoring systems.

We found that overall testing rates among patients arriving at the hospital was low (23\%), constituting a potential source of misclassification bias. Furthermore, testing was unequally distributed, with for instance $52 \%$

Table 3 Predictive value of dispatch and ambulance suspicion of Covid 19 among all patients, and tested patients only (April 14 August 31)

\begin{tabular}{|c|c|c|c|c|}
\hline & \multicolumn{2}{|c|}{ All patients $(n=6776)$} & \multicolumn{2}{|c|}{ Tested patients only $(n=2252)$} \\
\hline & Dispatch & Ambulance & Dispatch & Ambulance \\
\hline Apparent prevalence, \% & $16.8(15.9-17.7)$ & $24.9(23.9-25.9)$ & $31.9(30.0-33.9)$ & $49.3(47.2-51.4)$ \\
\hline True prevalence, $\%$ & $5.1(4.6-5.7)$ & $5.1(4.6-5.7)$ & $11.1(9.8-12.4)$ & $11.1(9.8-12.4)$ \\
\hline Sensitivity, \% & $75.9(71.0-80.3)$ & $82.2(77.8-86.1)$ & $71.1(65-76.6)$ & $79.1(73.5-84.0)$ \\
\hline Specificity, \% & $86.4(85.5-87.2)$ & $78.2(77.2-79.2)$ & $73.0(71.0-74.9)$ & $54.4(52.2-56.6)$ \\
\hline Positive predictive value, $\%$ & $23.1(20.7-25.7)$ & $16.9(15.2-18.8)$ & $24.7(21.5-28)$ & $17.7(15.5-20.1)$ \\
\hline Negative predictive value, $\%$ & $98.5(98.2-98.8)$ & $98.8(98.4-99.1)$ & $95.3(94.1-96.3)$ & $95.4(94.1-96.6)$ \\
\hline
\end{tabular}




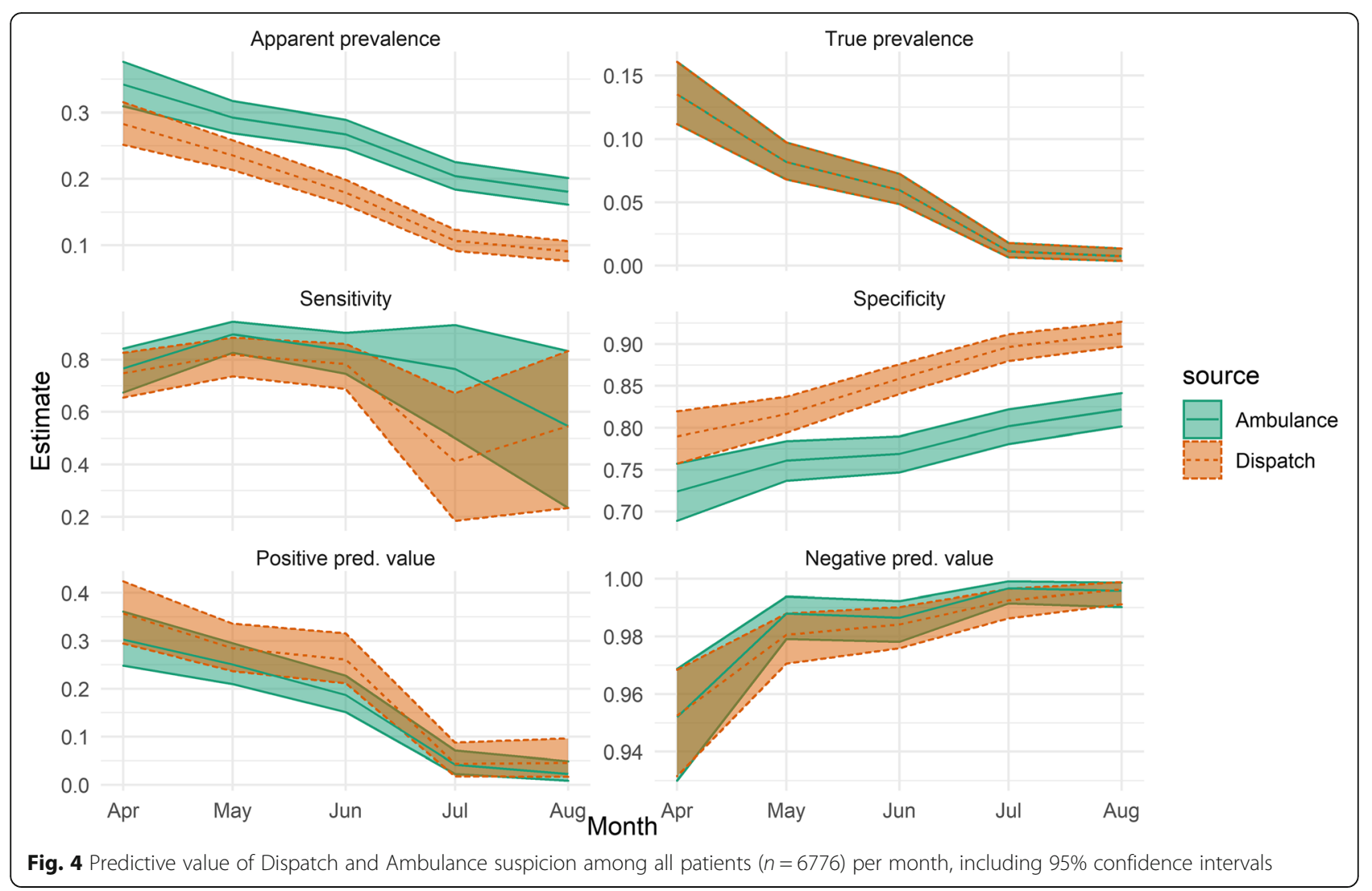

of patients admitted to the hospital tested for ongoing SARS-CoV-2 infection, but only $8 \%$ of patients discharged from the ED being tested. Some portion of these untested patients were in all likelihood asymptomatic or mildly symptomatic carriers of SARS-CoV-2 [18]. While false negative patients in this group are unlikely to have clinically severe Covid-19-related symptoms, they nonetheless represent a disease vector which must be identified and isolated to prevent contagion. Further misclassification may result from errors in the tests themselves, though the combined oro-nasopharyngeal sampling method employed in the region is considered to be perhaps the most sensitive available sampling approach for the diagnosis of ongoing SARS-CoV-2 infection [19].

To address the risk of misclassification-bias, we performed a sensitivity analysis investigating predictive value within only the cohort of patients whose SARSCoV-2 status was confirmed via PCR testing. Specificity was significantly lower in the sensitivity analysis, likely owing to the exclusion of a large number of negative cases. Estimates of sensitivity also tended to be lower in this analysis, but the difference could not be confirmed statistically. This loss of fidelity among patients identified via testing could be due to the inclusion of a larger proportion of patients with asymptomatic or mildly symptomatic SARS-CoV-2 infections, and/or the exclusion of clinically relevant positive cases identified by means other than PCR-testing.

The inclusion criteria for this study select for patients cared for through the typical prehospital care pathway of ambulance transport to the ED, and many patients referred to alternate forms of care by dispatchers and ambulance crews, as is common in the studied region [20], were not included owing to the lack of relevant outcome data for these patients. It may also be that some cases of Covid-19 were hospital-acquired, and that prehospital care providers could not have been expected to identify them. The data from this single-center study should be considered together with data collected in other settings to form a more general picture of the clinical impact of Covid-19 in the domain of prehospital care.

\section{Interpretation}

While the volume of Covid-19-related calls peaked in mid-April, the number of hospital episodes and admissions remained stable, owing to an increase in the number of patients directed by dispatchers and ambulance crews to non-ED destinations. In terms of pre-hospital acuity, Covid-19 patients had the lowest proportion of 
high priority (i.e., lights and sirens) dispatch responses of any comparison group (30\%), and similar levels of high priority transport compared to the baseline population (14\%). Expressed another way, the prevalence of Covid-19 did not differ between patients transported with a high- or low priority. Covid-19 patients were most likely to have signs of abnormal breathing (47\%), and to receive supplemental oxygen (36\%), but least likely to receive prehospital medications (28\%) compared with other patient cohorts. Outcomes for Covid-19 patients were substantially worse than any other cohort, with $16 \%$ of patients cared for in the ICU, and a 30-day mortality rate of $23 \%$.

It should be noted that prehospital priority was associated with patient outcomes in all comparison groups, as found in previous studies [21]. Within the cohort of Covid19-positive patients, the 30-day mortality rate of Covid-19 patients transported to the hospital with lights and sirens was for instance $40 \%$, compared with $21 \%$ for patients transported with a low priority. These findings thus suggest a discrepancy between prehospital prioritization and outcomes, and not a complete lack of association. This discrepancy could point to a difficulty in recognizing the early symptoms of severe Covid-19 infections, or to a more rapid deterioration among these patients after handover to definitive care.

Dispatch nurses were more selective in identifying suspected Covid-19 cases than their counterparts on the ambulance, with overall suspicion rates of $16 \%$ vs. $24 \%$. This resulted in a higher specificity ( $87 \%$ vs $79 \%)$, but a lower sensitivity ( $76 \%$ vs $82 \%)$. The negative predictive value of prehospital suspicion shifted from $95 \%$ at the beginning of the pandemic to $99 \%$ in the latter phases, likely due in large part to changes in the overall prevalence of Covid-19 in the population. A priori, we suspected that we might find an increasing trend in predictive value over time due to improved guidelines and care providers learning more about the presentation of Covid-19 patients. While we did observe a small initial uptick in sensitivity from April to May, we cannot confirm the existence of such an effect with a sufficient level of confidence based on these observational data, and this should be examined in further studies in other contexts.

While the level of predictive value found in this study is useful for the purposes of for instance system-level monitoring, the authors consider the level of sensitivity found here to fall short of the level required to rule out the need for isolation precautions on an individual basis. These results suggest that during periods of significant community transmission of Covid-19, provider suspicion alone is not sufficient to rule out the potential for contagion, and some level of isolation precautions should be adopted when interacting with all patients.
Our results demonstrate that roughly half of Covid-19 positive patients present with primary complaints associated with Covid-19 (infection, fever, upper airway complaints, or difficulty breathing), while significant numbers of Covid-19 patients present to prehospital care providers with non-typical complaints and may be difficult for care providers to identify. Simultaneously, only a minority of patients presenting with "typical" complaints were indeed later diagnosed with Covid-19. Previous studies have employed the documentation of a Covid-19-associated prehospital primary complaint as a proxy measure for the impact of Covid-19 at the system-level [8,9], and these results cast some doubt on the validity of this approach of gauging the true impact of Covid-19.

While patient vital signs demonstrated a limited capacity to detect the presence of SARS-CoV-2, they are likely to play a more substantial role in identifying severe cases with poor predicted outcomes [21-23]. The low overall testing rates and the preference towards testing higher-acuity patients found in this study entail a substantial risk for non-random misclassification-bias which would be imparted to models seeking to identify Covid19 positive patients within a general population where testing is not widespread or randomly distributed [24]. Approaches for mitigating such systematic misclassification bias in testing should be investigated in further research.

\section{Generalizability}

Our results were in line with a previous investigation by Fernandez et al. of the sensitivity of Covid-19 suspicion documented in free-text notes written by ambulance crews, but given the substantial difference in overall prevalence of Covid-19 between these studies (5\% here vs. $1 \%$ in Fernandez et al.) comparisons should be made only with caution, particularly with regards to specificity [11]. In examining the characteristics of Covid-19 patients presenting to the prehospital care system in this region, we found a $24 \%$ rate of 30 -day mortality, which is congruent with preliminary investigations of hospitalized patients diagnosed with Covid-19 in Denmark, Norway, and Italy $[23,25,26]$. The dispatch and ambulance personnel investigated here consisted of nurses with 3-4 years of formal education, which is a relatively high level of education by international standards. The idiosyncratic approach taken by Swedish public health authorities [27], the $23 \%$ overall testing rate, and $11 \%$ test positivity rate should also be considered when generalizing these results to other settings.

\section{Conclusion}

While prehospital suspicion was strongly indicative of hospital-confirmed Covid-19, based on the sensitivity identified in this study, prehospital suspicion should not 
be relied upon as a single factor to determine the need for isolation precautions on the ambulance or at the emergency department. The descriptive analysis revealed that Covid-19 patients presented with a diverse set of primary complaints. Despite a strikingly high rate of 30day mortality and ICU utilization, Covid-19 patients had a relatively low level of perceived prehospital acuity. The data provided may be used to improve guidelines for identifying Covid-19 patients in the prehospital setting.

\section{Abbreviations \\ Covid-19: Novel Coronavirus Disease 2019; SARS-CoV-2: Severe Acute Respiratory Syndrome Coronavirus 2; EMD: Emergency Medical Dispatch; PCR: (real-time,reverse transcription) polymerase chain reaction}

\section{Acknowledgements}

The authors thank the dispatch and ambulance nurses in the Uppsala region for generating the data we report, and for their tireless efforts to provide high-quality care throughout the ongoing pandemic.

\section{Authors' contributions}

DSp, HB and DSm conceived of and designed the study. DSp collected and analyzed the data, and drafted the manuscript. All authors contributed to revising the draft critically for important intellectual content and approved the final draft.

\section{Funding}

No external funding was used to finance this study. Open Access funding provided by Uppsala University.

\section{Availability of data and materials}

The datasets analyzed in the current study are available from the corresponding author and/or the Uppsala regional ambulance service (ambulanssjukvard@akademiska.se) on reasonable request.

\section{Ethics approval and consent to participate}

This study was approved by the Swedish Ethical Review Authority (dnr 202003093).

\section{Consent for publication}

Not Applicable.

\section{Competing interests}

The authors are employed by the regional ambulance service studied. The authors declare that they have no further competing interests.

Received: 5 November 2020 Accepted: 13 December 2020 Published online: 06 January 2021

\section{References}

1. Wiersinga WJ, Rhodes A, Cheng AC, Peacock SJ, Prescott HC. Pathophysiology, transmission, diagnosis, and treatment of coronavirus disease 2019 (COVID-19): a review. JAMA. 2020;324(8):782-93.

2. Rodriguez-Morales AJ, Cardona-Ospina JA, Gutiérrez-Ocampo E, VillamizarPeña R, Holguin-Rivera Y, Escalera-Antezana JP, et al. Clinical, laboratory and imaging features of COVID-19: a systematic review and meta-analysis. Travel Med Infect Dis. 2020;34:101623.

3. Pascarella G, Strumia A, Piliego C, Bruno F, Del Buono R, Costa F, et al. COVID-19 diagnosis and management: a comprehensive review. J Intern Med. 2020;288(2):192-206.

4. Chu DK, Akl EA, Duda S, Solo K, Yaacoub S, Schünemann HJ, et al. Physical distancing, face masks, and eye protection to prevent person-to-person transmission of SARS-CoV-2 and COVID-19: a systematic review and metaanalysis. Lancet. 2020;395(10242):1973-87.

5. Sanders JM, Monogue ML, Jodlowski TZ, Cutrell JB. Pharmacologic treatments for coronavirus disease 2019 (COVID-19): a review. JAMA. 2020; 323(18):1824-36

6. Vestergaard LS, Nielsen J, Richter L, Schmid D, Bustos N, Braeye T, et al. Excess all-cause mortality during the COVID-19 pandemic in Europe - preliminary pooled estimates from the EuroMOMO network, march to April 2020. Eurosurveillance. 2020;25(26):2001214.

7. Teixeira da Silva JA, Tsigaris P, Erfanmanesh M. Publishing volumes in major databases related to Covid-19. Scientometrics; 2020 [cited 2020 Sep 30]; Available from. https:/doi.org/10.1007/s11192-020-03675-3.

8. Jaffe E, Sonkin R, Strugo R, Zerath E. Evolution of emergency medical calls during a pandemic - An emergency medical service during the COVD-19 outbreak. Am J Emerg Med. 2020;S0735-6757:30527 [cited 2020 Oct 20]; Available from: http://www. sciencedirect.com/science/article/pii/S0735675720305271.

9. Fagoni N, Perone G, Villa GF, Celi S, Bera P, Sechi GM, et al. The Lombardy emergency medical system faced with COVID-19: the impact of out-ofhospital outbreak. Prehosp Emerg Care. 2020;0(0):1-7.

10. Katayama Y, Kiyohara K, Kitamura T, Hayashida S, Shimazu T. Influence of the COVD19 pandemic on an emergency medical service system: a population-based, descriptive study in Osaka, Japan. Acute Med Surg. 2020;7(1):e534.

11. Fernandez AR, Crowe RP, Bourn S, Matt SE, Brown AL, Hawthorn AB, et al. COVID-19 preliminary case series: characteristics of EMS encounters with linked hospital diagnoses. Prehosp Emerg Care. 2020;0(0):1-12.

12. Cleveland WS, Grosse E, Shyu WM. Local regression models. Chapter 8 in Statistical models in S (JM Chambers and TJ Hastie eds.). Pac Grove CA: Wadsworth BrooksCole; 1992. p. 608.

13. Davison AC, Hinkley DV. Bootstrap methods and their applications. Cambridge: Cambridge University Press; 1997. Available from: http://statumw.epfl.ch/davison/ BMA.

14. Collett D. Modelling binary data. Boca Raton: CRC press; 2002.

15. Nunes MS with contributions from T, Heuer C, Marshall J, Sanchez J, Thornton R, Reiczigel J, et al. epiR: Tools for the Analysis of Epidemiological Data. 2020. Available from: https://CRAN.R-project.org/package=epiR.

16. R Core Team. R: a language and environment for statistical computing. Vienna, Austria: R Foundation for statistical Computing; 2020. Available from: https:/unw.Rproject.org/.

17. von Elm E, Altman DG, Egger M, Pocock SJ, Gøtzsche PC, Vandenbroucke $J P$. The strengthening the reporting of observational studies in epidemiology (STROBE) statement: guidelines for reporting observational studies. J Clin Epidemiol. 2008;61(4):344-9.

18. Al-Sadeq DW, Nasrallah GK. The incidence of the novel coronavirus SARSCoV-2 among asymptomatic patients: a systematic review. Int J Infect Dis. 2020;98:372-80.

19. Diagnostic testing and screening for SARS-CoV-2. European Centre for Disease Prevention and Control. [cited 2020 Nov 2]. Available from: https:// www.ecdc.europa.eu/en/covid-19/latest-evidence/diagnostic-testing.

20. Spangler D, Edmark L, Winblad U, Colldén-Benneck J, Borg H, Blomberg H. Using trigger tools to identify triage errors by ambulance dispatch nurses in Sweden: an observational study. BMJ Open. 2020;10(3):e035004.

21. Spangler D, Hermansson T, Smekal D, Blomberg H. A validation of machine learning-based risk scores in the prehospital setting. Ashkenazi I, editor. PLOS ONE. 2019;14(12):e0226518.

22. Haimovich AD, Ravindra NG, Stoytchev S, Young HP, Wilson FP, van Dijk D, et al. Development and validation of the quick COVID-19 severity index: a prognostic tool for early clinical Decompensation. Ann Emerg Med. 2020;76(4):442-53.

23. Myrstad M, Ihle-Hansen H, Tveita AA, Andersen EL, Nygård S, Tveit A, et al. National Early Warning Score 2 (NEWS2) on admission predicts severe disease and in-hospital mortality from Covid-19 - a prospective cohort study. Scand J Trauma Resusc Emerg Med. 2020;28(1):66.

24. Gianfrancesco MA, Tamang S, Yazdany J, Schmajuk G. Potential biases in machine learning algorithms using electronic health record data. JAMA Intern Med. 2018:178(11):1544-7.

25. Giacomelli A, Ridolfo AL, Milazzo L, Oreni L, Bernacchia D, Siano M, et al. 30-day mortality in patients hospitalized with COVID-19 during the first wave of the Italian epidemic: a prospective cohort study. Pharmacol Res. 2020;158:104931.

26. Nersesjan V, Amiri M, Christensen HK, Benros ME, Kondziella D. 30-day mortality and morbidity in COVID-19 versus influenza: A population based study. 2020. Preprint at: https:/www.medrxiv.org/content/10.1101/2020.07.25.20162156v1.

27. Kamerlin SCL, Kasson PM. Managing Coronavirus Disease 2019 Spread With Voluntary Public Health Measures: Sweden as a Case Study for Pandemic Control. Clin Infect Dis. 2020; [cited 2020 Sep 30]; Available from: https:// academic.oup.com/cid/advance-article/doi/10.1093/cid/ciaa864/5866094.

\section{Publisher's Note}

Springer Nature remains neutral with regard to jurisdictional claims in published maps and institutional affiliations. 